\title{
Fitzpatrick Skin Type III
}

National Cancer Institute

\section{Source}

National Cancer Institute. Fitzpatrick Skin Type III. NCI Thesaurus. Code C74571.

Skin which is darker white and it sometimes burns when exposed to the sun. This type of skin can be seen in people with any hair or eye color. 